\title{
TOPOLOGY OPTIMIZATION OF ELASTO-PLASTIC STRUCTURES IN CONTACT
}

\author{
ANDRZEJ M. MYSLINSKI ${ }^{1}$ \\ ${ }^{1}$ Systems Research Institute \\ ul. Newelska 6, 01-447 Warsaw, Poland \\ myslinsk@ibspan.waw.pl
}

Key words: Structural optimization, Elasto-plastic contact problem, Regularization, Level set method

\begin{abstract}
This paper is concerned with the analysis as well as the numerical solution of the structural optimization problem for bilateral frictional contact problem where the static elasto-plastic material model with linear kinematic hardening rather than elastic material model is assumed. The displacement and stress of the bodies in elasto-plastic contact with a given friction are governed by the system of the coupled variational inequalities. In these problems usually very high stress appear along the surfaces in contact. It leads to wear or fatigue of the contacting surfaces. Therefore the aim of the topological optimization is to find such distribution of the material filling the body in contact to minimize the contact stress. Using von Mises yield function as well as the regularization and penalization techniques the original system of variational inequalities is transformed into the system of coupled nonlinear equations. The derivative of the cost functional with respect to the perturbation of the domain occupied by the body in contact is calculated using the material derivative method. Finite element method is used as the discretization method. In the numerical computations generalized Newton method is used to solve this contact problem. The level set method is used to describe and govern the evolution of the domain shape in the design space where the direction of the evolution is determined based on the calculated shape derivative. The results of computation are provided and discussed.
\end{abstract}

\section{INTRODUCTION}

Contact problems appear in many different fields of engineering including earthquake or civil engineering or machine dynamics. Therefore modeling of contact processes is an important topic which is currently still under investigation. Mathematical models describing the frictional or frictionless contact phenomenons between a deformable body and an obstacle, the so-called foundation, have been considered in many works $[17,18]$ where results dealing with the well-posedness of their solutions and/or numerical analysis as well as numerical simulation can be found.

The contact phenomenons between elasto-plastic bodies are reviewed in [6] where contact modeling approaches for different geometries and types of contact are described. Mathematical models of contact phenomenons for elasto-plastic materials are discussed in monographs [14, 17, 18]. In many applications the areas where the contact occurs are very small [12]. It implies that the transmitted contact force densities are usually rather big in the contact zone and it leads to plastic deformations. Therefore it is reasonable to consider an elasto-plastic rather than elastic model for the material. The high contact 
stress may lead to undesired vibrations or the degradation of surfaces of the contacting bodies as well as the deterioration of the working conditions for employees. The reduction of high contact stress or the obtaining the uniform distribution of this stress is the main aim of the topology or shape optimization problems for bodies in contact. The topology optimization consists in such distribution of the material filling the structure within the design domain to minimize the given cost functionals describing required features of the structure. Most research related to topology optimization has been concerned with linear elastic structures (see references in [12]). The amount of papers dealing with non-linear elastic structures or nonlinear mechanical behavior is limited. The ability to take into account elasto-plastic materials is of great interest of industrial engineers.

Structural optimization of elasto-plastic structures has been investigated, among others, in $[1,8,10$, $11,16]$. A major difficulty in shape or topology optimization of elasto-plastic problems as the pathdependent problems is the sensitivity analysis [10]. Therefore the original structural optimization problem has to be regularized either using the filter technique or different penalization techniques. Optimal control problems for elasto-plastic structures have been studied in a series of papers $[4,5]$ where primal and dual formulations as well as first order necessary optimality conditions are provided. Optimal control or structural optimization of contact problems for elasto-plastic materials have been considered in $[2,9,20]$.

The aim of this work is to analyze and to solve numerically the shape and topology optimization problem for two bodies in contact assuming static elasto-plastic material model with linear kinematic hardening rather than elastic material model. It extends the results in [13]. The optimization problem consists in finding such material distribution inside the domain occupied by the body in contact to minimize the
contact stress. In the paper penalization approach will be used to regularize the structural optimization
problem. Moreover the level set approach [15] will be used to solve it numerically. The small strain
plasticity material model is used [7]. The optimization problem is discretized using the finite el ment
method. The generalized Newton method is used to solve the discrete contact problem. Numerical results
are provided and discussed.

\section{Register for free att.tpsssymwossipedia.com to download the version without the watermark}

Consider a body occupying a bounded domain $\Omega \subset R^{d}, d=2,3$, with a Lipschitz continuous boundary $\Gamma$ (see Fig. 1). The boundary $\Gamma$ is divided into three open disjoint and measurable parts $\Gamma_{1}, \Gamma_{2}$ and $\Gamma_{3}$ such that meas $\left(\Gamma_{1}\right)>0$. The body is loaded by a given volume force of density $f_{1}=f_{1}(x)$ in domain $\Omega$ and a given surface traction of density $f_{2}=f_{2}(x)$ applied on the boundary $\Gamma_{2}$. The body is clamped along the boundary $\Gamma_{1}$, i.e., its displacement $u=\left(u_{1}, u_{2}\right), u=u(x), x \in \Gamma_{1}$, vanishes there. Along the boundary $\Gamma_{3}$ the body is assumed to be in bilateral frictional contact with the rigid foundation. The friction phenomenon is modeled by the Coulomb's law. The forces $f_{1}$ and $f_{2}$ are acting slow enough to neglect the inertial terms. The domain $\Omega$ is filled with a material undergoing under the loading of volume or boundary forces $f_{1}$ and $f_{2}$ elasto-plastic deformation with kinematic and isotropic hardening. In the elastic range it obeys Hooke's law $[2,7]$ defined by a fourth-order tensor $C$ such that, for any symmetric matrix $\zeta$,

$$
C \zeta=2 \lambda \zeta+\mu \operatorname{tr}(\zeta) I_{d},
$$

where $\lambda$ and $\mu$ are the Lamé constants and $I_{d}$ and $t r$ denote identity and trace operators, respectively. For $\mu>0$ and $d \lambda+2 \mu>0$ the elasticity tensor $C \in L^{\infty}(\Omega)$ is assumed to be symmetric, uniformly bounded 
and coercive $[2,7]$. Let us also introduce the Cauchy stress tensor $\sigma$ and the linearized strain tensor $\varepsilon$ $[2,7]$, respectively:

$$
\sigma=\sigma(u)=\left\{\sigma_{i j}\right\}_{i=1}^{d}, \text { and } \varepsilon=\varepsilon(u)=\frac{1}{2}\left(u_{i, j}+u_{j, i}\right), i, j=1, \ldots, d, u_{i, j}=\frac{\partial u_{i}}{\partial x_{j}} .
$$

The summation convention over repeated indices $[2,7]$ is used throughout the paper. The divergence operator $\operatorname{div}(\sigma)$ of a second order tensor $\sigma$ is defined as $\operatorname{div}(\sigma)=\left\{\sigma_{i j, j}\right\}, \quad \sigma_{i j, j}=\frac{\partial \sigma_{i j}}{\partial x_{j}}, \quad i, j=1, \ldots, d$. We assume the plastic deformation of the material is governed by the additive small strain plasticity model $[7,18]$. In this model the material strain $\varepsilon$ is sum of the elastic strain $\varepsilon^{e}$ and the plastic strain $\varepsilon^{p}$, i.e.,

$$
\varepsilon(u)=\varepsilon^{e}(u)+\varepsilon^{p}(u),
$$

and the stress tensor satisfies

$$
\sigma(u)=C \varepsilon^{e}(u)=C\left(\varepsilon(u)-\varepsilon^{p}(u)\right), \text { and } \varepsilon(u)=C^{-1} \sigma(u)+\varepsilon^{p}(u) .
$$

The plastic deformation with the hardening phenomenon is governed by the generalized plastic strain $\left(\varepsilon^{p}, \xi\right)$ and the generalized plastic stresses $(\sigma, \chi)$. The variable $\xi$ represents a set of scalar or tensorial internal variables capturing the feature of hardening behavior [7]. The back stress $\chi$ denotes an internal force arising during the hardening and causing the translation of the initial yield surface. The back stress $\chi$ and the internal variable $\xi$ are related by [7]

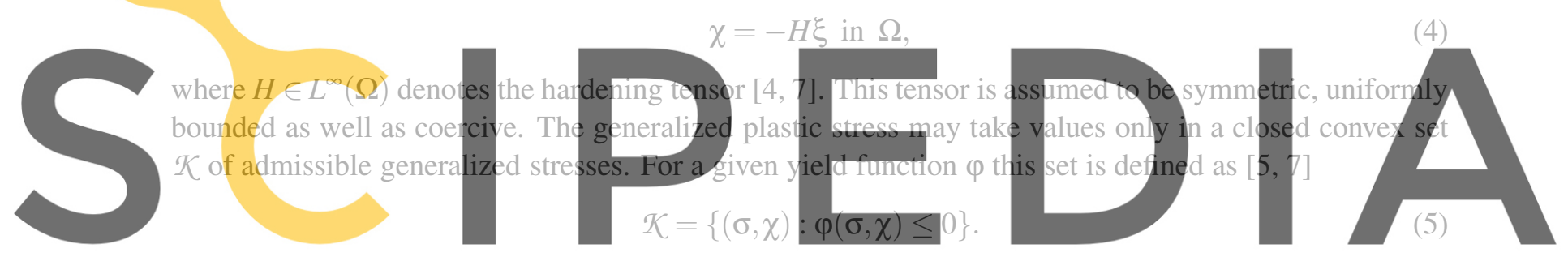

The elastic region of the loaded material corresponds to $\varphi(\sigma, \chi)<0$, i.e., the interior of the cone $\mathcal{K}$.

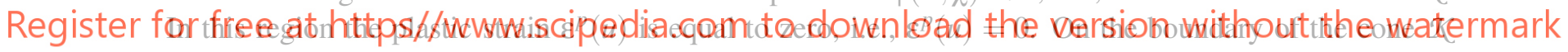
called the yield surface, where $\varphi(\sigma, \chi)=0$, the plastic strain can be nonzero. The evolution of the plastic strain $\varepsilon^{p}$ and the internal variable $\xi$ is governed by the associative flow rule $[7,10]$ stating that the rates of plastic strain and the internal variable belong to the normal cone $N_{K}$ to the set $K$ at a point $(\sigma, \chi)$. For the smooth yield function it implies the existence of the nonnegative scalar $\tilde{\lambda}$ and the Kuhn-Tucker complementarity conditions relating $\tilde{\lambda}$ and the function $\varphi$ [7]

$$
\left(\dot{\varepsilon}^{p}, \dot{\xi}\right)=\tilde{\lambda} \nabla \varphi(\sigma, \chi), \text { and } \tilde{\lambda} \geq 0, \varphi(\sigma, \chi) \leq 0, \tilde{\lambda} \varphi(\sigma, \chi)=0 .
$$

where the dot above the symbol denotes the derivative with respect the time variable, i.e., $\dot{\xi}=\frac{\partial \xi}{\partial t}$. Condition (6) is also consistency condition according to which for $\varphi=0$, plastic loading takes place if $\dot{\varphi}=0$ while unloading appears if $\dot{\varphi} \leq 0$. The flow rule can also be described using maximal plastic work principle for the generalized stresses [7]

$$
\begin{array}{r}
\varepsilon(u(x)): \sigma(x)-\sigma(x): C^{-1}: \sigma(x)-\chi(x): H^{-1}: \chi(x)= \\
\max _{(q, \eta) \in \mathcal{K}}\left\{\varepsilon(u(x)): \sigma(x)-\sigma(x): C^{-1}: q(x)-\chi(x): H^{-1}: \eta(x)\right\} .
\end{array}
$$


Let us denote by $R^{d \times d}$ the space of real $d \times d$ matrices. We shall consider the following contact problem: find the generalized stress field $(\sigma, \chi): \Omega \rightarrow R^{d \times d}$, the displacement field $u: \Omega \rightarrow R^{d}$, the generalized strain field $\left(\varepsilon^{p}, \xi\right): \Omega \rightarrow R^{d \times d} \times R^{d}$ satisfying plasticity conditions (2) - (7) as well as

$$
\begin{array}{r}
\operatorname{div} \sigma+f_{1}=0 \text { in } \Omega, \\
u=0 \text { on } \Gamma_{1} \text { and } \sigma_{v}=f_{2} \text { on } \Gamma_{2}, \\
u_{v}=0,\left|\sigma_{\tau}\right| \leq \mu_{f}\left|\sigma_{v}\right| \text { on } \Gamma_{3}, \\
\left|\sigma_{\tau}\right|<\mu_{f}\left|\sigma_{v}\right| \Rightarrow \dot{u}_{\tau}=0 \text { on } \Gamma_{3}, \\
\left|\sigma_{\tau}\right|=\mu_{f}\left|\sigma_{v}\right| \Rightarrow \exists \bar{\lambda} \geq 0, \dot{u}_{\tau}=-\bar{\lambda} \sigma_{\tau} \text { on } \Gamma_{3} .
\end{array}
$$

For the unit outward normal vector $v$ to the boundary $\Gamma$ normal and tangential components of the displacement field $u$ are denoted $[7,18]$ by $u_{v}=u \cdot v=u_{i} \cdot v_{i}, i=1, \ldots, d$, and by $u_{\tau}=u-u_{v} v$, respectively. Similarly normal and tangential components of the stress field $\sigma$ are denoted by $\sigma_{v}=\sigma v \cdot v$ and by $\sigma_{\tau}=\sigma v-\sigma_{v} v$, respectively. $\mu_{f}$ is the friction coefficient and $|\cdot|$ denotes the Euclidean norm. System (2)-(12) governs the elasto - plastic bilateral contact problem with Coulomb friction.

\subsection{Variational problem}

For the sake of sensitivity analysis let us introduce the variational formulation of this contact problem. We shall use the dual rather than primal variational formulation of the contact problem (2)-(12) with von Mises yield function $\varphi$ i than on the generalized load condition to ensure the function $\varphi_{M}$ has the foll
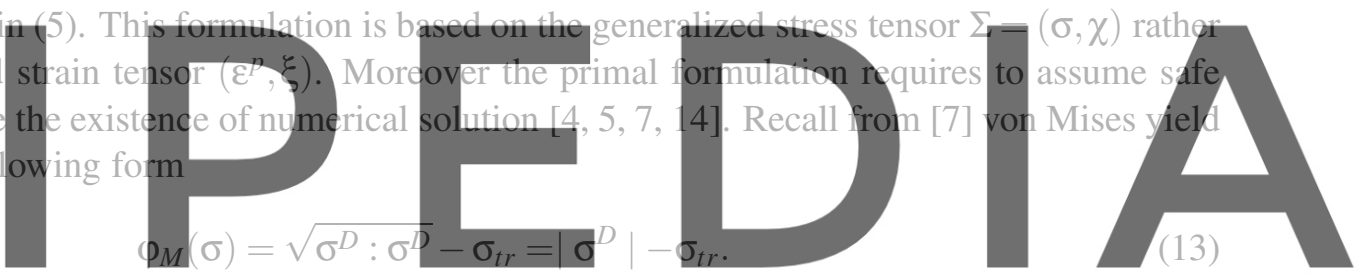

The constant $\sigma_{t r}>0$ and $\sigma_{D}: \sigma_{B}$ denote the material yield stress and the product of fourth order tensors

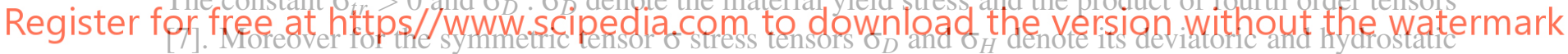
components, respectively,

$$
\sigma^{D} \stackrel{d f}{=} \sigma-\frac{i r(\sigma)}{d} I_{d} \quad \sigma^{H} \stackrel{d f}{=} \frac{i r(\sigma)}{d} I_{d}
$$

Therefore using (13) the set of admissible generalized stresses (5) takes the form

$$
K_{M}=\left\{(\tau, \eta) \in S \times S:\left|\tau^{D}+\eta^{D}\right|-k_{2} \gamma \leq \sigma_{t r}\right\},
$$

where $k_{2} \geq 0$ is the isotropic hardening parameter and $\gamma$ is a scalar determining expansion of the yield surface in the isotropic hardening or equivalent plastic strain [7]. For the sake of simplicity we assume linear hardening only, i.e., $k_{2}=0$. We denote by $V$ and $S$ the space for displacements and the space for stresses as well as back stresses, respectively:

$$
V=\left\{u \in H^{1}\left(\Omega ; R^{d}\right): u=0 \text { on } \Gamma_{1}\right\} \text { and } S=L^{2}\left(\Omega ; R_{s y m}^{d \times d}\right) .
$$

The subspace $R_{\text {sym }}^{d \times d} \subset R^{d \times d}$ denotes the subspace of symmetric matrices or symmetric second-order tensors. Here $L^{2}\left(\Omega ; R^{d}\right)$ and $H^{1}\left(\Omega ; R^{d}\right)$ denote the space of the square integrable functions as well as the 
space of the square integrable functions and their first derivatives in domain $\Omega$ taking values in $R^{d}$. For details see [7]. The set of admissible displacements is denoted by

$$
K_{C}=\left\{u \in V: u_{v}=0 \text { on } \Gamma_{3}\right\} .
$$

By $C^{-1}$ and $H^{-1}$ we denote the compliance tensor, i.e., the inverse tensor to the elasticity tensor $C$ and the inverse tensor to the hardening tensor $H$, respectively. Tensors $C^{-1}$ and $H^{-1}$ are assumed to be symmetric, uniformly bounded and coercive [7]. For $\sigma \in S$ and $v \in V$ we define the linear form $l: V^{\prime} \times V \rightarrow R$ defined as

$$
l(v)=\int_{\Omega} f_{1} \cdot v d x+\int_{\Gamma_{2}} f_{2} \cdot v d s,
$$

where $V^{\prime}$ denotes the space dual to the space $V$ [7]. The friction functional $j_{c}(\cdot): V \rightarrow R$ is defined as follows

$$
j_{c}(v)=\int_{\Gamma_{3}} \mu_{f}\left|\sigma_{v}(u)\right|\left|\nu_{\tau}\right| d s .
$$

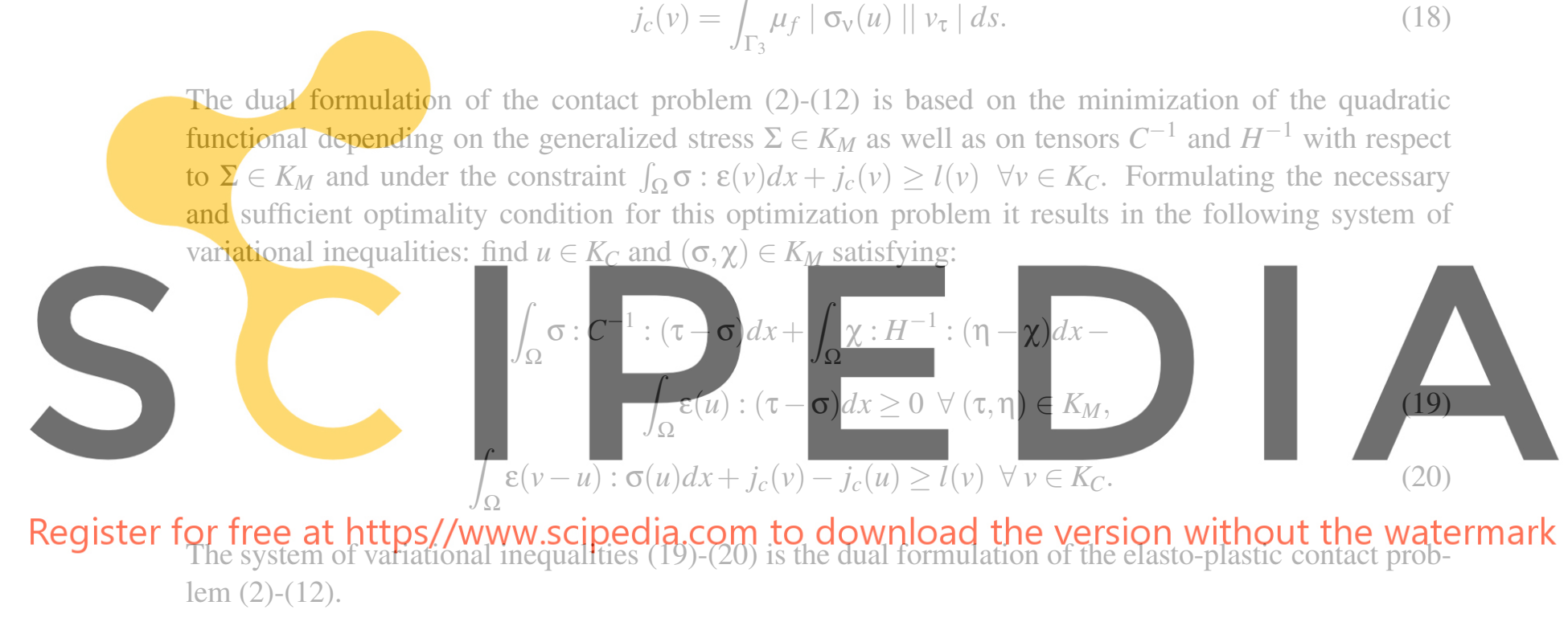

\subsection{Contact problem regularization}

Elasto-plastic contact problem is governed by the system of coupled variational inequalities (19)-(20). Applying penalization and regularization techniques we shall transform it into the system of nonlinear equations. It allows sensitivity analysis as well as numerical solution of the optimization problem. First we shall deal with the plasticity conditions.

\subsubsection{Penalization of plasticity conditions}

Remark, the first two terms of the inequality (19) describe the projection of the generalized stress tensor $\Sigma$ on the admissible set $K_{M}$ [10]. For von Mises yield function (13) the orthogonal projection operator 
$P_{K_{M}}(\Sigma)$ of the generalized stress tensor $\Sigma$ on the set $K_{M}$ with respect to the scalar product in $S \times S$ is expressed as $[4,10]$

$$
P_{K_{M}}(\Sigma)=\Sigma-\frac{1}{2} \max \left(0,\left|\sigma_{D}+\chi_{D}\right|-\sigma_{t r}\right) \frac{1}{\left|\sigma_{D}+\chi_{D}\right|}\left(\begin{array}{c}
\sigma_{D}+\chi_{D} \\
\sigma_{D}+\chi_{D}
\end{array}\right) .
$$

Therefore using the projection operator $P_{K_{M}}$ the constraint $\Sigma \in K_{M}$ can be expressed as the quadratic penalty term equal to the $S \times S$ norm of the difference between $\Sigma$ and $P_{K_{M}}(\Sigma)$ added to the dual energy functional [4] and depending on a given real number penalization parameter $\tilde{\alpha}>0$. This penalization is based on the Moreau-Yosida approximation of the indicator function of the set $K_{M}$ of the admissible generalized stresses and is elasto-viscoplastic approximation of the elasto-plastic problem (19)-(20) [4]. It allows to consider the inequality (19) on the whole space rather than on the cone $K_{M}$. The modification of the dual energy cost functional generates also a new system of the optimality conditions. The necessary and sufficient optimality condition for this penalized optimization problem results in the following system of variational equations and inequalities: for $\tilde{\alpha}>0$ find $u_{\tilde{\alpha}} \in K_{C}$ and $\left(\sigma_{\tilde{\alpha}}, \chi_{\tilde{\alpha}}\right) \in S \times S$ satisfying:

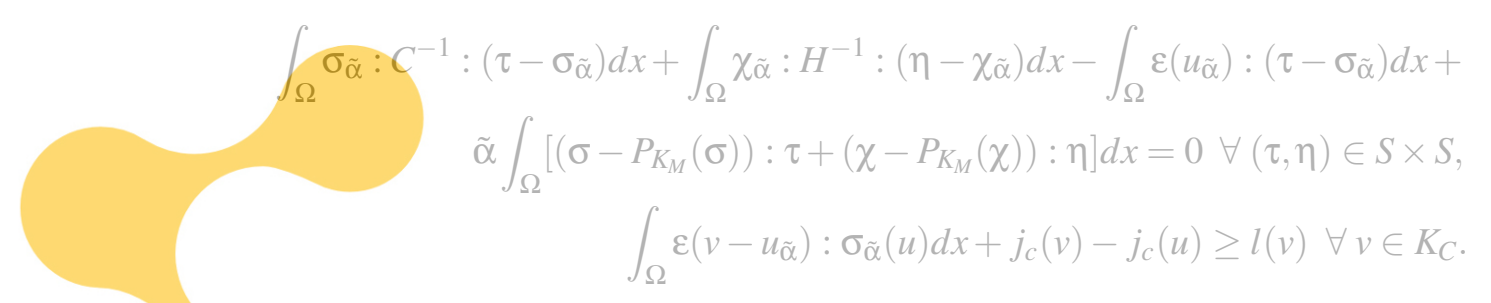

In the formula (21) appears the non-differentiable mapping $x \rightarrow \max (0, x)$. Therefore the erator (21) is also non-d. we regularize this mapp the function $\max (0, \cdot)$. We $\alpha>0$. Function $\tilde{f}_{\alpha}(\cdot)$ h
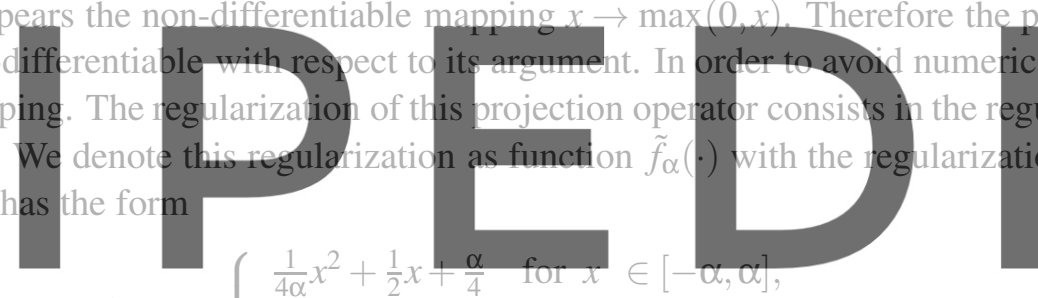

Using (21) and (24) the regularized projection operator $P_{K_{M}}^{\alpha}(\Sigma)$ of the operator (21) depending on parameter $\alpha$ can be written as

$$
P_{K_{M}}^{\alpha}(\Sigma)=\Sigma-\tilde{f}_{\alpha}\left(\left|\sigma_{D}+\chi_{D}\right|-\sigma_{t r}\right) \frac{1}{\left|\sigma_{D}+\chi_{D}\right|}\left(\begin{array}{c}
\sigma_{D}+\chi_{D} \\
\sigma_{D}+\chi_{D}
\end{array}\right) .
$$

Taking into account the regularized projection operator (25) rather than non-smooth operator (21) in the system (22)-(23) we obtain the system with the regularized plasticity conditions: find $u_{\tilde{\alpha}, \alpha} \in V$ and $\left(\sigma_{\tilde{\alpha}, \alpha}, \chi_{\tilde{\alpha}, \alpha}\right) \in S \times S$ satisfying:

$$
\begin{array}{r}
\int_{\Omega} \sigma_{\tilde{\alpha}, \alpha}: C^{-1}:\left(\tau-\sigma_{\tilde{\alpha}, \alpha}\right) d x+\int_{\Omega} \chi_{\tilde{\alpha}, \alpha}: H^{-1}:\left(\eta-\chi_{\tilde{\alpha}, \alpha}\right) d x-\int_{\Omega} \varepsilon\left(u_{\tilde{\alpha}, \alpha}\right):\left(\tau-\sigma_{\tilde{\alpha}, \alpha}\right) d x+ \\
\tilde{\alpha} \int_{\Omega}\left[\left(\sigma_{\tilde{\alpha}, \alpha}-P_{K_{M}}\left(\sigma_{\tilde{\alpha}, \alpha}\right)\right): \tau+\left(\chi_{\tilde{\alpha}, \alpha}-P_{K_{M}}\left(\chi_{\tilde{\alpha}, \alpha}\right)\right): \eta\right] d x=0 \quad \forall(\tau, \eta) \in S \times S, \\
\int_{\Omega} \varepsilon\left(v-u_{\tilde{\alpha}, \alpha}\right): \sigma_{\tilde{\alpha}, \alpha}(u) d x+j_{c}(v)-j_{c}\left(u_{\tilde{\alpha}, \alpha}\right) \geq l(v) \forall v \in K_{C} .
\end{array}
$$

Remark, the equation (26) is formulated on the whole space $S \times S$ rather than on the set $K_{M}$. 


\subsubsection{Regularization of friction conditions}

Let us assume Tresca friction model, i.e., $\left|\sigma_{v}(u)\right|=h, h>0$ is given. Therefore in the inequality (27) the frictional functional (18) is the non-smooth term due to the $|\cdot|: R^{d} \rightarrow R$. We approximate this function by the smooth function $\varphi_{\rho}: R^{d} \rightarrow R$ dependent on the regularization parameter $\rho>0$. In general this function is assumed to be a convex non-negative $C^{2}$ function such that $\varphi_{\rho}(0)=0$. As a function $\varphi_{\rho}$ we choose $\varphi_{\rho}(v)=\sqrt{\rho^{2}+v^{2}}-\rho$. Using this regularizing function let us denote by $\left\{j_{c}^{\rho}(\cdot)\right\}_{\rho}$ a family of convex contact friction functionals $j_{c}^{\rho}(\cdot): V \rightarrow R_{+}$of class $C^{2}$ depending on the regularization parameter $\rho>0$, i.e.,

$$
j_{c}^{\rho}(v)=\int_{\Gamma_{3}} \mu_{f} h \varphi_{\rho}\left(v_{\tau}\right) d s \forall v \in V .
$$

The gradient $\nabla_{v} j_{c}^{\rho}(\cdot): V \rightarrow V^{\prime}$ of functional (28) with respect to the argument is equal to:

$$
\int_{\Gamma_{3}} \nabla_{\nu} j_{c}^{\rho}(u) v d s=\int_{\Gamma_{3}} \mu f h \frac{d \varphi_{\rho}(u)}{d u} v d s \quad \forall v \in V
$$

Using (28) the inequality (27) is approximated by the sequence of equations depending on parameters $(\tilde{\alpha}, \alpha, \rho)$

$$
\int_{\Omega} \varepsilon\left(v-u_{\tilde{\alpha}, \alpha, \rho}\right): \sigma_{\tilde{\alpha}, \alpha, \rho}(u) d x+\int_{\Gamma_{3}} \nabla_{v} j_{c}^{\rho}\left(u_{\tilde{\alpha}, \alpha, \rho}\right) v d s=<l, v>\forall v \in V
$$

For the sake of simplicity let us denote by $\beta=(\tilde{\alpha}, \alpha, \rho)>0$ a real parameter. Using the approximations (22)-(23), (26)-(27) and $(1)$

nonlinear equations: for of nonlinear equations:
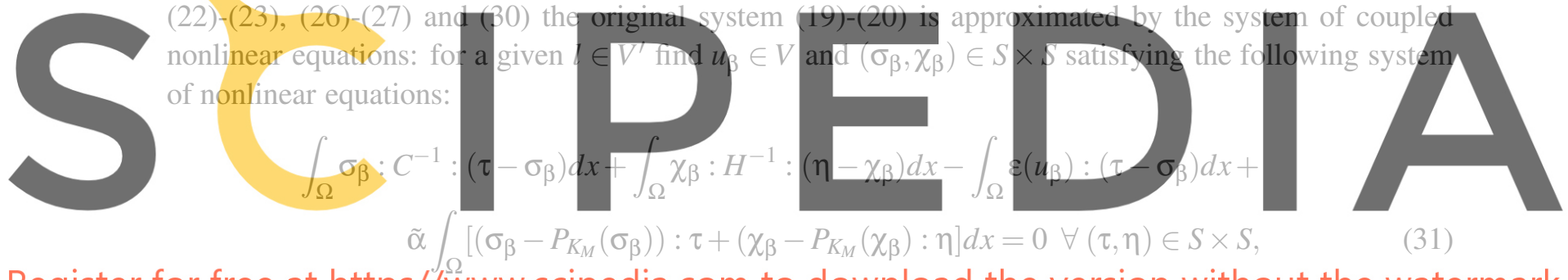

\section{TOPOLOGY OPTIMIZATION PROBLEM}

Let us formulate the optimization problem for the state system (31)-(32). The objective functional $J(\cdot)$ : $V \rightarrow R$ is assumed to depend on the solution $u(\Omega)$ to the state system (31)-(32) in the domain $\Omega$. In general this functional is sum of domain and/or boundary integrals, i.e.,

$$
J(u(\Omega))=\int_{\Omega} \psi(u) d x+\int_{\Gamma} \tilde{\psi}(u) d s,
$$

where the integrand functions $\psi: R^{d} \rightarrow R$ and $\tilde{\psi}: R^{d} \rightarrow R$ depend also on the solution $u=u(\Omega)$ to the state system (31)-(32). These integrand functions are assumed smooth enough and their first derivatives are bounded for every $u$. Each admissible domain $\Omega$ is assumed to be contained [3] in open and bounded hold-all domain $D \subset R^{d}$. Therefore the set $\mathcal{U}_{a d}$ of the admissible domains is written as

$$
\mathcal{U}_{a d}=\left\{\Omega \subset R^{d}: \Omega \subset D, \Omega \text { is suitable regular and satisfies imposed constraints } g(\Omega) \leq 0\right\},
$$


where $g$ is a given function. Consider the following shape optimization problem: find domain $\Omega^{\star} \in \mathcal{U}_{a d}$ minimizing the objective functional (33) on the set of admissible domains (34), i.e.,

$$
J\left(u\left(\Omega^{\star}\right)\right)=\min _{\Omega \in \mathcal{U}} J(u(\Omega)),
$$

where $(u(\Omega), \sigma(\Omega), \chi(\Omega))$ is the solution to the state system (31)-(32) in the domain $\Omega$. In order to find minimum of the objective functional (33) first we need to calculate its derivative with respect to the design variable, i.e., its shape derivative. We shall calculate it using the Lagrangian as well as material derivative frameworks $[3,19]$. Since the regularization mapping $(25)$ is a smooth Lipschitz function it is also point-wise differentiable. The solution $u$ to the state problem (31)-(32) is more regular, i.e., it belongs to the space $L^{2+\delta}(\Omega)$ for $\delta>0$. It implies that $\sigma(u)$ is also more regular. Therefore the mapping (25) is strongly differentiable. Denote by $P_{K_{M}}(\Sigma)^{\prime}$ the derivative of $P_{K_{M}}(\Sigma)$ with respect to $\Sigma$. Recall the adjoint variables $(z, p, q) \in V \times S \times S$ associated with the state variables $(u, \sigma, \chi) \in V \times S \times S$ can be retrieved by differentiating the Lagrangian with respect to $u$ and $(\sigma, \chi)$ in the directions $v$ and $(\tau, \eta)$. It gives the system of adjoint equations satisfied by the variables $(z, p, q)$ :

$$
\begin{aligned}
& \int_{\Omega} \frac{d \psi(u)}{d u} v d x+\int_{\Gamma} \frac{d \tilde{\psi}(u)}{d u} v d s-\int_{\Omega} \varepsilon\left(v_{\beta}\right):\left(p-\sigma_{\beta}\right) d x+ \\
& \int_{\Omega} \varepsilon\left(z-v_{\beta}\right): \sigma_{\beta}(v) d x+\int_{\Gamma_{2}} \nabla_{v} j_{c}^{\rho}\left(w, v_{\beta}\right) z d s=0 \quad \forall v \in V,
\end{aligned}
$$

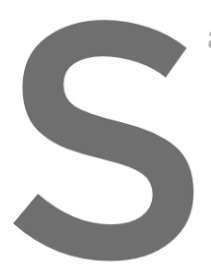

as well as
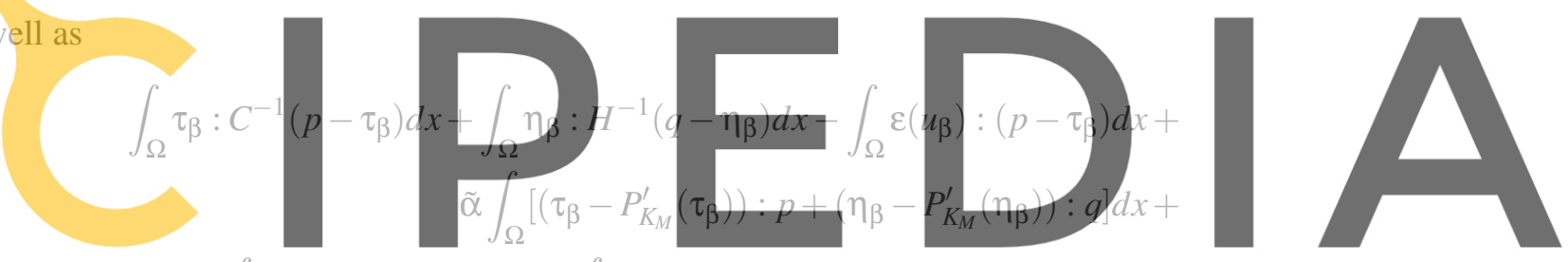

$\int \varepsilon\left(z-u_{\beta}\right): \tau_{\beta}(u) d x+\int \nabla_{v} j_{c}^{p}\left(\tau(w), u_{\beta}\right) z d s=0 \forall(\tau, \eta) \in S \times S$.

(37)

Register for free at https/1/www.scipedia.com to download the version without the watermark

Therefore using (36)-(37) as well as formulas for shape derivatives of domain and boundary integrals $([3,19])$ we obtain the shape derivative of the cost functional $(33)$ in the direction of a velocity field $\zeta$ as equal to

$$
\begin{array}{r}
J^{\prime}(\Omega)(\zeta)=\int_{\Gamma}\left(\psi(u)-f_{1} z\right) \zeta v d x+\int_{\Gamma}\left(H_{m c} \tilde{\psi}(u)+\partial_{v} \tilde{\psi}\right) \zeta v d s-\int_{\Gamma}\left(H_{m c} f_{2} z+\partial_{v} f_{2} z\right) \zeta v d s+ \\
\int_{\Gamma} \sigma_{\beta}: C^{-1} p \zeta v d s+\int_{\Gamma} \chi_{\beta}: H^{-1} q \zeta v d s-\int_{\Gamma} \varepsilon\left(u_{\beta}\right): p \zeta v d s+\tilde{\alpha} \int_{\Gamma}\left[\left(\tilde{f}_{\alpha}\left(\sigma_{\beta}\right)\right): p+\left(\tilde{f}_{\alpha}\left(\chi_{\beta}\right): q\right] \zeta v d x+\right. \\
\int_{\Gamma} \varepsilon(z): \sigma_{\beta}(u) \zeta v d s+\int_{\Gamma_{2}}\left[H_{m c} \nabla_{v} j_{c}^{\rho}\left(u_{\beta}\right) z+\partial_{v} \nabla_{v} j_{c}^{\rho}\left(u_{\beta}\right) z\right] \zeta v d s
\end{array}
$$

where $(u, \sigma, \chi)$ and $(z, p, q)$ are solutions to the state system (31)-(32) and the adjoint system (36)-(37), respectively. In (38) $H_{m c}$ denotes mean curvature of the boundary $\Gamma$. 


\section{NUMERICAL METHODS}

We use the level set function [15] to define and to update the shapes of the sequence of the admissible domains $\Omega$. All admissible shapes $\Omega$ are contained in a hold-all open bounded domain $D \subset R^{d}$. The level set function $\Psi(\cdot): R^{d+1} \rightarrow R$ is defined as:

$$
\begin{cases}\Psi(x)<0 & \text { if } x \in \Omega, \\ \Psi(x)=0 & \text { if } x \in \Gamma \cap D, \\ \Psi(x)>0 & \text { if } x \notin \Omega .\end{cases}
$$

The boundary $\Gamma$ of domain $\Omega$ is located as a set of points in $D$ where the level set function $\Psi(x)$ is equal to 0 . Inside (outside) domain $\Omega$ this function takes negative (positive) values. The domain and boundary integrals of function $f$ are transfered [15] from domain $\Omega$ into domain $D$ using Heaviside function $H$ and Dirac function $\delta$, respectively,

$$
\int_{\Omega} f(x) d x=\int_{D} f(x)\left(1-H(\Psi(x)) d x, \quad \int_{\Gamma} f(x) d s=\int_{D} f(x) \delta(\Psi(x))|\nabla \Psi(x)| d x\right.
$$

The one-dimensional Heaviside and Dirac functions are given by:

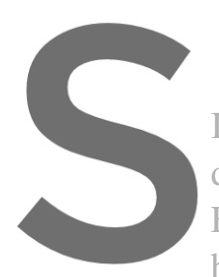

$$
H(\Psi)= \begin{cases}0 & \text { if } \Psi \leq 0 \\ 1 & \text { if } \Psi>0\end{cases}
$$

\section{In computations these}

domain $\Omega_{0}$. During the

Each domain $\Omega_{i}$ is charac

be associated with fictitious time
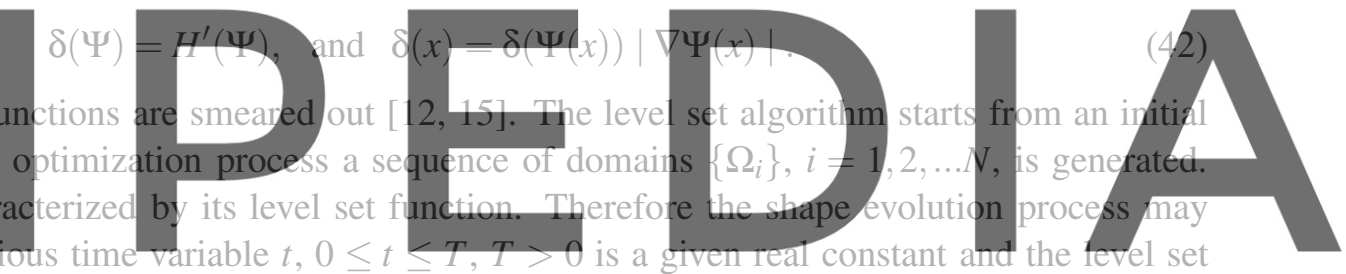

function is dependent on space and time variables, i.e., $\Psi=\Psi(t, x)$. In classical setting the evolution of

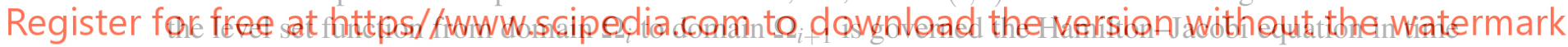
interval $[0, T]$

$$
\frac{\partial \Psi}{\partial t}+\zeta|\nabla \Psi|=0, \Psi(0, x)=\Psi_{0}(x) \text { in } D,
$$

where function $\Psi_{0}(x)$ determining the shape of the initial domain $\Omega_{0}$ is given. The normal velocity of the domain boundary $\zeta(x)$ is identified with the shape derivative (38) of the objective functional (33) in the direction normal to the boundary $\Gamma$. Since the shape derivative is given as boundary integral the normal velocity $\zeta$ has to be extended on the whole computational domain $D[3,15]$. This extension is usually associated with the regularization of velocity $\zeta$. The equation (43) is solved usually by an explicit second order upwind scheme on a Cartesian grid of design domain $D$ with Neumann boundary conditions. In order to ensure the stability of this scheme the time step has to satisfy CFL condition relating the length of time and space discretization steps [15]. Since during the iteration process the level set may become too flat or too steep in order to regularize it periodic reinitialization of the level set function is performed. For other types of the level set methods such as binary or piecewise constant see [12, 15].

Both the state and the adjoint boundary value problems (31)-(32) as well as (36)-(37) are discretized using bilinear quadrilateral finite elements. Ersatz material approach $[3,10,11]$ is used to avoid meshing 
problems with the shapes of domains $\Omega$ and singularity of the stiffness matrix. This approach consists in filling the domain $D \backslash \Omega$ with a weak material characterized by law value of Young modulus and mimicking void. The discretized state and adjoint equations are solved numerically using generalized Newton method [20].

\section{NUMERICAL EXAMPLE}

Shape optimization problem for a body occupying two-dimensional domain $\Omega \subset R^{2}$ in bilateral contact with the rigid foundation has been solved numerically in Matlab environment. The functional (33) with $\psi(u)=0$ and $\tilde{\psi}(u)=\sigma(u) \cdot v \phi \cdot v$ is chosen as the objective functional where $\phi$ is a given function. The aim of the shape optimization problem is to reduce the contact stress. The constraint function $g(u)$ in (34) is equal to volume constraint, i.e., $g(u)=\operatorname{Vol}(u)=\int_{\Omega} d x-V_{0}$. The domain $\Omega \subset R^{2}$ is chosen as follows (see Fig. 1)

$$
\Omega=\left\{\left(x_{1}, x_{2}\right) \in R^{2}: 0 \leq x_{1} \leq 8 \wedge 0<v\left(x_{1}\right) \leq x_{2} \leq 4\right\},
$$

where the function $v\left(x_{1}\right)=0.125 \cdot\left(x_{1}-4\right)^{2}$ describes the boundary $\Gamma_{3}$. The boundary $\Gamma$ of the domain $\Omega$ is divided into three disjoint pieces: $\Gamma_{1}=\left\{\left(x_{1}, x_{2}\right) \in R^{2}: x_{1}=0,8 \wedge 0<v\left(x_{1}\right) \leq x_{2} \leq 4\right\}, \Gamma_{2}=$ $\left\{\left(x_{1}, x_{2}\right) \in R^{2}: 0 \leq x_{1} \leq 8 \wedge x_{2}=4\right\}, \Gamma_{3}=\left\{\left(x_{1}, x_{2}\right) \in R^{2}: 0 \leq x_{1} \leq 8 \wedge v\left(x_{1}\right)=x_{2}\right\}$.

Domain $\Omega$ is filled with the solid material characterized by the Young moduli $E_{1}=10 \cdot E_{0}$ and with weak material in the form of distributed voids characterized by the Young moduli $E_{2}=.1 \cdot E_{0}, E_{0}=2.1 \cdot 10^{5}$ $\mathrm{MPa}$ (see Fig. 2). The Poisson's ratio is equa to $v=.3$. The shear and dilation moduli are equal to $8 \cdot 10^{4}$
$\mathrm{MPa}$ and $1,1 \cdot 10^{5} \mathrm{MPa}$, respectively. The yield stress $\sigma_{t r}=367,4 \mathrm{MPa}$. The hardening parameters are
equal to $k_{1}=1 \cdot 10^{5} \mathrm{MPa}$ and $k_{2}=0 \mathrm{MPa}$. The body is loaded by the boundary traction $f_{2}=-6.5 \cdot 10$
$\mathrm{~N}$ along the boundary $F_{1}$, the body forec $f_{1}=0 \mathrm{in}$ domain $\Omega$. The hold-all domain $D$ is a rectangle
$[0,8] \times[0,4]$. This domain is divided into $80 \times 40$ grid. Fig. $\beta$ displays the obtained optimal topo/ogy. The areas of weak material appear in the central part of the domain $\Omega$ and close to the clamped boundary

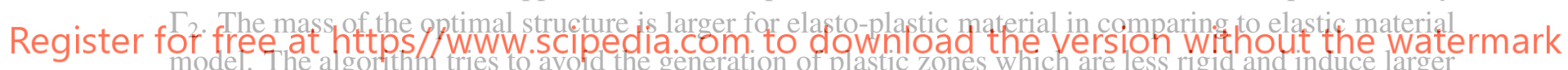

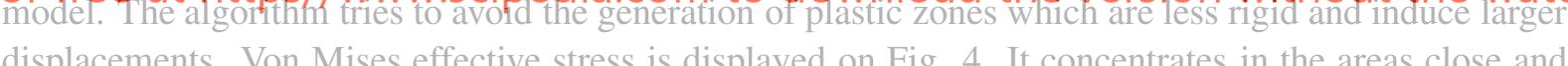
above the contact zone. The obtained normal contact stress is almost constant along the optimal shape boundary and has been significantly reduced comparing to the initial one.

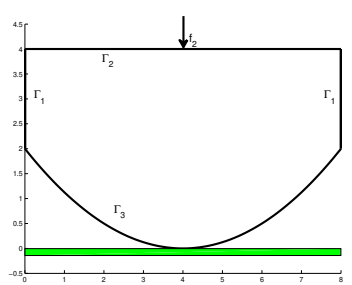

Figure 1: Contact between domain $\Omega$ and the rigid foundation.

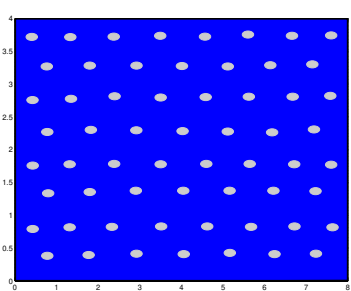

Figure 2: Initial computational domain $D$. 


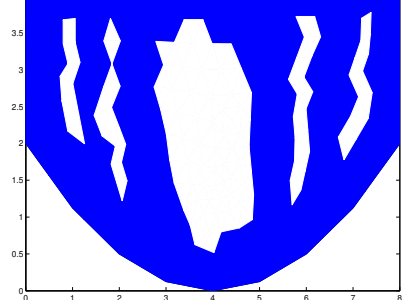

Figure 3: Optimal topology domain $\Omega^{\star}$.

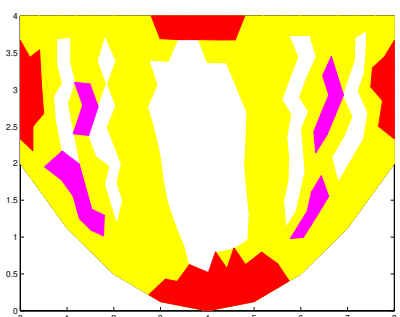

Figure 4: von Mises stress distribution

\section{CONCLUSIONS}

The obtained results indicate that presented approach based on the application of the level set technique can be applied to solve numerically a topology optimization problem for bodies in bilateral frictional contact where nonlinear small strain elasto-plastic with linear kinematic hardening material model rather than elastic material model is used. It allows to formulate necessary optimality conditions for this type of nonlinear problems and it is capable of finding topologies that generates minimum contact stress. This approach is flexible and can be extended to solve other shape or topology optimization problems for structures governed by nonlinear equations.

\section{REFERENCES}

[1] Blachowski, B., Tauzowski, P., Lógó, J. Yield limited optimal topology design of elastoplastic structures, Struct. Multidisc. Optim., (2020) 61:19531976.

[2] Capatina, A., Timofte, C. Boundary optimal control for quasistatic bilateral frictional contact problems, Nonlinear Analysis (2014) 94:84-99.

[3] Delfour, M. C., Zolesio, J.P. Shapes and Geometries: Metrics, Analysis, Differential Calculus, and Optimization, Second Edition, Advances in Design and Control series (2011):22, SIAM, 2011.

[4] De los Reyes, J. C., Herzog, R. and Meyer, C. Optimal control of static elastoplasticity in primal formulation, SIAM J. Control Optim., (2016) 54:3016-3039.

[5] De los Reyes, J. C. On the optimal control of some nonsmooth distributed parameter systems arising in mechanics, GAMM-Mitt. (2017) 40:268-286.

[6] Ghaednia, H., Wang, X., Saha, S., Xu, Y., Sharma, A., Jackson, R. L. A Review of Elastic-Plastic Contact Mechanics, Appl. Mech. Rev. (2017) 69:060804 1-30.

[7] Han, W., Reddy, B.D. Plasticity. Mathematical Theory and Numerical Analysis. 2nd edition, Springer, New York, 2013.

[8] Li, L., Zhang, G., Khandelwal, K. Topology optimization of energy absorbing structures with maximum damage constraint, International Journal for Numerical Methods in Engineering, (2017) 112:737-775.

[9] Kim, N.H., Choi, K.K and Chen, J. S. Shape design sensitivity analysis and optimization of 
elasto-plasticity with frictional contact, AIAA Journal, (2000) 38:1742-1753.

[10] Maury, A., Allaire, G., Jouve, F. Elasto-plastic shape optimization using level set method, SIAM J. on Control Optim. (2018) 56:556-581.

[11] Maute, K., Schwarz S., Ramm, E. Adaptive topology optimization of elastoplastic structures, Structural Optimization (1998)15:81-91.

[12] Myśliński, A. Piecewise constant level set method for topology optimization of unilateral contact problems, Advances in Engineering Software (2015) 80:25-32.

[13] Myśliński, A. Topology Optimization of Elasto-Plastic Contact Problems, Proceedings of the 4th Polish Congress of Mechanics and 23rd International Conference on Computer Methods in Mechanics PCM-CMM-2019, Kraków, Poland, September 8-12, 2019, Eds. Witold Cecot, Marek Kozien, Wojciech Lisowski, Kinga Nalepka, Jerzy Pamin, Waclaw Reczek and Blazej Skoczen, AIP Conference Proceedings 2239, 020031 (2020).

[14] Necas, J., Hlavácek, I. Mathematical Theory of Elastic and Elasto-Plastic Bodies, Elsevier, 2002.

[15] Osher, S., Fedkiw, R. Level set methods and dynamic implicit surfaces, Applied Mathematical Sciences series, (2003):153, Springer-Verlag, New York, 2003.

[16] Rohan, E., Whiteman, J.R. Shape optimization of elasto-plastic structures and continua, Comput. Methods Appl. Mech. Engrg. (2000) 187:261-288.

[17] Sofonea, M., Matei, A. Mathematical Models in Contact Mechanics, London Mathematical Society, Lecture Notes Series 398, Cambridge University Press, 2012

[18] Sofonea, M., Migórski, S. Variational-Hemivariational Inequalities with Applications, CRC Press, Boca Rayton, FL, 2018.

[19] Sokołowski, J., Zolesio, J.P. Introduction to shape optimization, Springer series in Computational Mathematics 16, Springer, Berlin, (1992).

[20] Werner, S., Stingl, M., Leugering, G. Model-based control of dynamic frictional contact problems using the example of hot rolling, Comput. Methods Appl. Mech. Engrg. (2017) 319:442-471. 